\title{
How joint line orientation affects the amount of bone resected in mechanically aligned TKA: a retrospective clinical-radiographic correlational study
}

\section{Zhiwei Wang}

Beijing Chaoyang Hospital https://orcid.org/0000-0002-5668-075X

Yang Yu

Beijing Chaoyang Hospital

Liang Wen

Beijing Chaoyang Hospital

Desi Ma

Beijing Chaoyang Hospital

Tiebing Qu ( $\nabla$ qtb@medmail.com.cn )

China Rehabilitation Research Center https://orcid.org/0000-0003-2800-5404

\section{Research article}

Keywords: Joint line, Bone resection, Osteoarthritis, Alignment, Total knee arthroplasty

Posted Date: January 7th, 2021

DOI: https://doi.org/10.21203/rs.3.rs-38300/v2

License: (c) (1) This work is licensed under a Creative Commons Attribution 4.0 International License. Read Full License 


\section{Abstract}

Background: Discrepancies in bone resection between the medial and lateral compartments is very common in total knee replacement (TKA) when mechanical alignment (MA) is used. The purpose of this study was to explore whether and how joint line orientation affects the gross amount of bone resected in mechanically aligned TKA.

Methods: A total of 112 patients (137 knees) diagnosed with osteoarthritis (OA) were included. Bone resection simulations were conducted in the coronal view using full-length weight-bearing radiographs per the technical parameters of MA, and the feasibility of simulated osteotomy was verified via intraoperative caliper measurement. Correlation and regression analyses were conducted comparing the amount of bone resected and various parameters, including hip-knee-ankle (HKA) angle, mechanical lateral distal femoral angle (mLDFA), joint line congruency angle (JLCA), and medial proximal tibial angle (MPTA). Moreover, any intraoperative bone resection adjustments that were made were retrospectively collected using a medical record query system.

Results: All knees in the current case series could be artificially divided into 4 subgroups: 84 varus knees $(61.3 \%)$ with valgus femurs in subgroup 1, 32 varus knees (23.4\%) with varus femurs in subgroup 2, 14 valgus knees $(10.2 \%)$ with varus tibias in subgroup 3 , and 7 valgus knees $(5.1 \%)$ with valgus tibias in subgroup 4. Simulated bone resection thickness was highly consistent with intraoperative measurements. MPTA and mLDFA were positively correlated with the amount of bone resected in subgroup 1. The regression equation was: Maximum resection thickness $(\mathrm{mm})=0.556^{\star} \mathrm{mLDFA}(\mathrm{deg}$.) + $0.098 \star M P T A$ (deg.) $-39.74 ; R^{2}=0.808$. Similarly, intraoperative bone resection adjustments also showed a correlation with mLDFA in subgroup 1.

Conclusions: Deformities in knee OA can be divided into 4 subgroups based on the alignment of extremities and the joint line orientation. When TKA is performed in varus knees with valgus femurs using conventional instruments and mechanical alignment techniques, the amount of bone resected in the coronal plane demonstrates a linear relationship with the patient's mLDFA.

\section{Background}

Restoration of the neutral mechanical axis of the knee is considered the target alignment in total knee arthroplasty (TKA) when the mechanical alignment technique is applied. The postoperative neutral alignment of the lower extremity with a margin of error of $3^{\circ}$ has been shown to distribute contact forces evenly and improve the long-term survivorship of implants [1-4]. Both the femoral and tibial components should be implanted perpendicular to the mechanical axis of the lower extremity $[5,1]$.

According to the published literature $[6,7]$, in patients with knee osteoarthritis (OA) undergoing TKA, the thicknesses of the resected portions of the medial and lateral condyles of the distal femur are significantly different. Similarly, the thicknesses of the resected portions of the medial and lateral tibial plateaus are different as well [8]. As a consequence, the extension gap (EG) can differ significantly 
between the medial and lateral compartments, despite narrowing or widening of the overall EG. It is for this reason that the distal femoral and tibial cutting blocks in nearly all conventional TKA instruments provide at least $+/-2 \mathrm{~mm}$ parallel pin holes for resection level adjustments.

As mentioned above, the EG is highly variable in actual surgical operations, especially when using conventional instruments. Sometimes an uneven EG is an iatrogenic consequence of accidental mishandling by surgeons. The natural inclination of the knee joint line, however, may also play an important role in such clinical scenarios. The hypothesis of the current study is that joint line inclination could affect the thickness of the primary bone resection in TKA.

\section{Patients And Methods}

This study is a correlational analysis using retrospective radiologic measurements and supposed bone resection in the EG. Various joint line orientation parameters in OA knees were measured on anteroposterior full-length weight-bearing roentgenogram. Bone cut simulations were performed to evaluate the gross bone loss. The relationship between various measurement parameters and the supposed EG space was investigated to judge the effects of joint line orientation on the EG in TKA.

\section{Patients}

A total of 112 consecutive patients (patient demographics are shown in Table 1) diagnosed with OA receiving primary TKA (137 knees) in our hospital between July 2017 and July 2019 were included in the current study. The inclusion criteria were patients with Grade III-IV Kellgren-Lawrence knee OA, and fulllength weight-bearing radiographs of both lower extremities in which the projection of the patella was centered between the femoral condyles were obtained. The exclusion criteria included fixed subluxation or dislocation of the patella, congenital lower extremity deformity, history of injury or operation around the knee joint, significant corrosion of subchondral bone or significant deformities compared with the contralateral knee, $>10$ degrees of flexion contracture, inflammatory arthritis, and abnormal rotation (patellar edge beyond the contour of femoral condyle) of the lower extremities due to technical aspects of imaging.

\section{Radiographic measurements and bone cut simulation}

Acquisition of standard full-length weight bearing radiograph. In frontal plane, AP view, the full-length weight bearing radiographs were obtained under following conditions: Definium 8000 digital radiographic system (GE, MA, USA) with 1.8 meters of exposure distance, $80 \mathrm{Kv}$ of voltage, $400 \mathrm{~mA}$ of electric current and auto exposure mode was used in this study. Patients usually were instructed to stand with the feet together and patella faced forward. In case of tibial torsion, rotate the foot to orient the patella forward. Hip, knee and ankle joints were exposed separately, and full-leg weight bearing radiographs were automatically spliced and synthesized by the system. 
Determination of anatomical landmarks and joint line parameters. Picture archiving and communication system (PACS) was used to generate radiological measurements in this study. The determination of anatomical landmarks and various joint orientation angles was performed according to the method described by Paley et al[9]. The center of the femoral head was identified using the Mose circles approach. The center of the distal femur, tibial plateau and ankle joint were defined using the top of the femoral notch, the center of the tibial spines and the mid-width of the talus, respectively. The mechanical axes of the femur and tibia were subsequently determined by connecting the above anatomical points. The distal femoral joint line was defined as the line tangential to the medial and lateral femoral condyles, and the proximal tibial joint line was similarly defined as the line tangential to the concave aspect of the subchondral line of the tibial plateau.

Measurement of joint orientation parameters. The hip-knee-ankle (HKA) angle was defined as the angle between the femoral and tibial mechanical axes. The HKA angle was defined as a negative value in the varus knee and a positive value in the valgus knee. The mechanical lateral distal femoral angle (mLDFA) was defined as the lateral angle formed by the femoral mechanical axis and the distal femoral joint line. The joint line congruency angle (JLCA) was defined as the angle formed by the distal femoral joint line and the proximal tibial joint line. Medial convergence of the JLCA was defined as a negative value, and a lateral JLCA was defined as a positive value. The medial proximal tibial angle (MPTA) was defined as the medial angle between the mechanical tibial axis and the proximal tibial joint line.

Measurement of bone cut simulation in the frontal plane. The bone cut simulation was carried out via the mechanical alignment (MA) technique using PACS. On the femoral side, a line (Line A) that was both tangential to the most distal point of the femur (either the medial or lateral condyle) and perpendicular to the femoral mechanical axis was drawn. Then, another line (Line B) parallel to Line A and $9 \mathrm{~mm}$ proximal to Line A was drawn. Finally, the distances from Line B to the most distal part of the medial and lateral femoral condyles were measured and recorded as the medial femoral resection thickness and lateral femoral resection thickness, respectively (Fig. 1a). On the tibial side, a line (Line C) that was both tangential to the most prominent articular surface (either the medial or lateral plateau) and perpendicular to the tibial mechanical axis was drawn. Similarly, another line (Line D) parallel to Line C and $10 \mathrm{~mm}$ distal to Line $C$ was set. The distances from Line $D$ to the two points on the medial and lateral concave aspects of the tibial plateau were measured as the medial tibial resection thickness and lateral tibial resection thickness, respectively (Fig. 1b). The magnification of the radiographs was 1.05. All image measurements were performed by two independent physicians (YY, DM). After the reliability test was performed, the mean values were utilized in the subsequent statistical analysis.

Estimate of total bone resection. The medial resection thickness was calculated as the sum of the medial femoral resection thickness and the medial tibial resection thickness, as for the lateral resection thickness. Regardless of the medial or lateral resection thickness, only the larger value (hereafter referred to as the "max. resection") was used in the following calculations. This algorithm is required using the MA technique. In clinical scenarios, to ensure postoperative lower extremity alignment, the distal femoral and proximal tibial resections must be perpendicular to their individual mechanical axes. As a result, 
discrepancies between medial and lateral resection amounts are very common. If the EG on one side is too narrow due to less bone being resected, ligament release, rather than an additional parallel resection, is usually necessary to achieve EG balance.

\section{Surgical procedure and intraoperative verification}

Operations were performed by two senior attending surgeons (ZW, LW). Gemini MK-Il prostheses (Link, Hamburger, Germany) were used in this study, of which 131 were the cruciate-retaining type and 6 were the posterior stabilized type. Conventional instruments were used. The distal femoral resection was conducted perpendicular to the femoral mechanical axis [10]. After the valgus resection angle was set accordingly, a 9-mm thick resection area was determined based on the most prominent femoral condyle. Tibial resection was conducted perpendicular to the tibial mechanical axis, and the resection level was estimated using the stylus, $10 \mathrm{~mm}$ from the less worn plateau or the most prominent side of the lateral plateau. The EG was subsequently preliminarily estimated. Measured resection techniques were used in the current study. When the bone preparation was completed, any necessary ligament balancing was performed before the components were cemented.

As this study is retrospective, we only collected the intraoperative resection data of the 10 patients finally included in this study in order to verify the accuracy of the bone cut simulation. The resected bone pieces from the distal femoral condyles and proximal tibia were calipered and recorded for comparison with the preoperative radiographic measurement data (Fig. 2 a, b).

\section{Retrospective collection of resection data}

The original medical records of all included patients were retrospectively analyzed. The patient's surgical records were reviewed for specific elements for further analysis, including whether the patient received an extra cut in the distal femur or proximal tibia, whether a thicker liner was used, and whether an augment was used or thicker bone cement was applied beneath the femoral component to balance the flexionextension gap.

\section{Statistical analysis}

The data were summarized using mean (SD) or median values. The normal distribution of the data was tested via the Shapiro-Wilk test. Correlation analysis was used to investigate the relationship between the max. resection amount and various joint orientation parameters. Linear regression analysis was conducted to identify predictive factors of bone cut amount. Reliability analysis using the intraclass correlation coefficient (ICC) was conducted for image measurements and intraoperative resection verification. All statistical tests were performed using SPSS statistical software (version 22; SPSS, Inc., Chicago, IL, USA). A P-value of $<0.05$ was considered statistically significant. All plots in this study were generated using R (4.0.0, R Core Team (2020), Vienna, Austria) and the ggplot2 package (3.2.1, Wickham (2020), New York, US). 


\section{Results}

Patient demographics and various joint orientation parameters are shown in Table 1. Interestingly, among the 137 knees included in this study, the MPTAs in all varus knees were less than 90 degrees, and the mLDFAs in all valgus knees were less than 90 degrees. This finding could be understood to mean the following: when the MA technique is used in TKA for bone preparation, lateral tibial plateau resections are always thicker than medial resections in varus knees, while the resection thicknesses of medial femoral condyles is always greater than that of lateral condyles in valgus knees.

Varus knees can be divided into 2 subgroups based on the mLDFA value: subgroup 1, with varus knees with $\mathrm{mLDFA}<90^{\circ}$, and subgroup 2 , with varus knees with $\mathrm{mLDFA}>90^{\circ}$. In the same way, valgus knee can be divided into 2 subgroups based on the MPTA value: subgroup 3 , with valgus knees with MPTA $<90^{\circ}$, and subgroup 4, with valgus knees with MPTA $>90^{\circ}$ (Table 1) (Fig. 3). These groups were formed prior to conducting further analysis because in subgroup 2 , the resection level of the distal femur and tibial plateau refer to the lateral femoral condyle and lateral tibial plateau, respectively. After resection, the lateral compartment has acquired enough space to accommodate the components, and the medial compartment is usually narrower than the lateral compartment. Next, osteophyte removal and extensive soft tissue release are required to achieve EG balance. Similarly, in subgroup 4, extensive lateral soft tissue release is often needed to achieve EG balance after the primary bone cut. Therefore, data from subgroups 2 and 4 were not included in the subsequent analysis because the max. resection in both groups was constant.

Radiographic measurement data from subgroups 1 and 3 were included in the subsequent analysis. Key parameters, including HKA, mLDFA, JLCA, MTPA, and max. resection, were tested for normality. The results showed that only mLDFA and max. resection values in subgroup 1 were normally distributed, with $p=0.003$ and $p=0.004$, respectively.

To investigate the relationship between the resection amount and various joint orientation parameters, Spearman's correlation coefficients were calculated. The results showed that mLDFA and MPTA were positively correlated with max. resection amount in subgroup 1, with correlation coefficients of 0.931 and 0.304 and $p$ values of $<0.001$ and 0.005 , respectively. Linear regression was subsequently conducted using mLDFA and MPTA to investigate which parameter had a better predictive capacity. The regression equation was derived as follows: $y=0.556 x_{1}+0.098 x_{2}-39.74$, where $y$ represents the max. resection and $x_{1}$ and $x_{2}$ represent mLDFA and MPTA, respectively, with an $R^{2}$ of 0.808 (Fig. 4). On the other hand, Spearman's correlation analysis showed that only MPTA had a weak positive correlation with max. resection in subgroup 3 , with a correlation coefficient of 0.009 . The regression equation is $y=0.415 x-$ $19.493, R^{2}=0.617$ (Fig. 5).

To determine the reliability of the radiographic measurements and intraoperative verification of the bone cut simulation, reliability analysis was conducted. The ICCs of HKA, mLDFA, JLCA and MPTA were 0.999 (95\% Cl: 0.999 1), 0.962 (95\% Cl: 0.947 0.972), 0.998 (95\% Cl: 0.998 0.999), and 0.997 (95\% Cl: 
0.996 0.998), respectively. The ICC of intraoperative verification was 0.984 (95\% Cl: 0.969 0.991). Statistical analysis showed that the image measurements had high consistency between the two observers, and the simulated bone resection in PACS was highly consistent with intraoperative measurement.

A retrospective surgical record survey showed that $70.8 \%$ of patients did not receive further adjustments (0) following the primary bone resection, while other patients received various intraoperative adjustments, including $2 \mathrm{~mm}$ of further tibial resection (+2t), $2 \mathrm{~mm}$ of further distal femoral resection (+2f), and both $(+4)$, as well as a $2 \mathrm{~mm}$ thicker liner $(-2 \mathrm{t})$. Very rarely, approximately $2 \mathrm{~mm}$ of additional cement was applied between the distal femur and the component to address a relatively wider EG (-2f) (Table 2). No other types of adjustments were found in the review of the surgical records of this case series.

To investigate whether there was a correlation between intraoperative adjustment of the primary resection and joint line orientation, grouped scatter plots were generated using surgical records from subgroups 1 and 3. In subgroup 1, mLDFA was used as an independent variable because of its greater impact (Fig. 6a), while in group 3, MPTA was used as an independent variable (Fig. 6b). In cases of varus knees with mLDFAs of $\angle 90^{\circ}$, as the mLDFA decreases, the chances of a bone recut increases, irrespective of whether the extra bone cut occurs on the femoral or tibial side. Conversely, as the mLDFA approaches $90^{\circ}$, the chances of using thicker liners slightly increases. On the other hand, in the case of valgus knees with MPTAs $<90^{\circ}$, although the MPTA and intraoperative resection adjustment present similar trends, this relationship is less convincing due to the smaller number of cases.

\section{Discussion}

The goal of this study was to investigate the correlation between various anatomical parameters and the extent of bone resection in TKA when MA and conventional instruments are used. The safety and reliability of the MA technique has been proven for decades [1, 11], so it is considered the "gold standard" for TKA $[12,13]$. Regrettably, MA cannot fully take into account the patient's natural lower extremity alignment and joint line orientation[14]. In fact, significant variation in the overall coronal limb alignment and femoral and tibial joint lines has been proven to exist in osteoarthritic knees [15]. Therefore, we speculate that the interaction of the above two factors leads to inaccurate primary bone resections when TKA is performed using conventional instruments.

Based on the classic MA philosophy, the distal femoral and proximal tibial resection should be perpendicular to the mechanical axis of the femur and tibia, respectively. The resection thickness should be equal to the corresponding component thickness to guarantee proper soft tissue tension in the full range of motion without extra soft tissue release $[16,17]$. In most patients, however, neither the femoral condyles nor the tibial plateaus are perpendicular to the mechanical axis. As a consequence, the resection thicknesses of the femoral condyles is not always the same, as is the case with the tibial plateau [18].

In femoral bone preparation, when a conventional instrument is used, a valgus alignment jig, together with an intramedullary rod, is usually used to determine the orientation of the distal femoral cut. 
Theoretically, the distal femoral resection thickness is determined using an adjustable jig, but the actual resection thickness of each condyle is determined based on which condyle contacts the jig first. In most cases, the medial part of the jig contacts the articular surface first; consequently, the resection thickness of the medial femoral condyle is usually equal to the desired thickness, while the resection thickness of the lateral femoral condyle is usually less than that of the medial condyle. On the other hand, the resection thickness of the medial tibial plateau is usually less than that of the lateral plateau, especially in most knee OA cases with neutral or varus alignment. Therefore, the amount of bone removed is generally less than that to be replaced with components.

Although the discrepancy in medial and lateral EGs could be corrected with osteophyte removal or ligament release, a relatively narrower EG necessitates additional distal femur cut to accommodate the implants, and a wider EG indicates undesirable bone loss, a thicker polyethylene insert or distal femoral augments might be required to achieve extension stability and appropriate joint line levels. The reason for this may be a combination of MA operative principles, high variation in joint line orientation, constitutional varus or valgus alignment, and possible surgical technical error.

Researchers have begun to consider the correlation between anatomical alignment parameters of the lower extremities and different TKA alignment techniques. In one study [19], Hirschmann et al. combined individual's HKA, femoral mechanical angle (supplementary angle of the mLDFA) and tibial mechanical angle (same as MPTA) to divide the knee joint into a group of "functional knee phenotypes." These phenotypes were matched with the target of different TKA alignment techniques, and it was found that only $5.6 \%$ of males and $3.6 \%$ of females met the MA alignment target. In another study [20], Lin et al. investigated the relative parameters of lower limb alignment in relation to one another in a young nonosteoarthritic population, and all the subjects were categorized based on HKA, mLDFA and MPTA. In Lin's study, all subjects could be classified into one of five types of alignment: 2 types of neutral alignment, 2 types of varus alignment and 1 type of valgus alignment. The coronal neutral alignments in Hirschmann's and Lin's studies were defined as " $-1.5^{\circ}<\mathrm{HKA}<1.5^{\circ}$ " and " $-3^{\circ}<\mathrm{HKA}<3^{\circ}$ ", respectively, rather than HKA=0 .

To enhance the clinical relevance of the current correlational analysis, all subjects included in our study were patients diagnosed with knee $\mathrm{OA}$, and all patients were divided into the valgus or varus knee groups based on whether their HKA was greater than $0^{\circ}$. Through the measurement of alignment parameters, all varus knees were found to have MPTAs less than $90^{\circ}$, and all valgus knees had mLDFAs less than $90^{\circ}$. These findings are consistent with those of previously published literature, and the major contributors to valgus knee and varus knee are valgus of the distal femur[21] and varus of the proximal tibia[7], respectively.

In our case series, patients in the varus knee group were divided into 2 subgroups based on whether their mLDFA was less than $90^{\circ}$, while those in the valgus knee group were similarly divided with reference to the MPTA. Usually, the coronal deformities in subgroups 2 and 4 are more serious than those in the other two subgroups because both the femoral and tibial bony variations contribute to the coronal deformity. Lateral bowing of the femoral shaft may be an important factor leading to the relatively larger mLDFA in 
subgroup 2[22, 23]. According to the requirements of the MA technique, bone resection must be perpendicular to the femoral and tibial mechanical axes. Therefore, when determining the level of the bone resection, the lateral condyle and lateral tibial plateau should be used as references in varus knees in subgroup 2. Consequently, an appropriate gap can be easily achieved in the lateral compartment, but extensive ligament release is inevitable in the medial compartment because of the reduced extent of bone removal and intrinsically tight medial collateral ligament [24]. A similar situation occurs in valgus knees in subgroup 4, for which the bone resection levels are determined by the medial condyle and medial tibial plateau. Extensive soft tissue release or even constrained prostheses can be used to achieve acceptable mediolateral balance.

To the best of our knowledge, no prior study has focused on the relationship between joint line orientation and the extent of bone resection performed. One study found a significant correlation between the leg axis and the optimal tibial resection thickness, and the optimal resection thickness in valgus knees was significantly less than that in neutral or varus knees; however, no joint line orientation parameters were considered[8]. Inconsistent with the results of the above study, the amount of bone resection was not related to the HKA in this study. The reason for this difference may be because joint line orientation parameters and simulated resection data for only subgroups 1 and 3 were included in this correlational analysis.

Patients in subgroup 1 had varus knees with valgus femurs and accounted for the majority of all included patients (61.3\%). Correlational analysis showed that both MPTA and mLDFA were positively correlated with the extent of bone resection. The results of the subsequent regression analysis showed that mLDFA, rather than MPTA, can have a significant effect on the amount of bone resection, with regression coefficients of 0.556 and 0.098 , respectively. This was truly an unexpected finding. The results of the regression analysis revealed that for every $2^{\circ}$ increase in mLDFA, the amount of bone resection increases by approximately $1 \mathrm{~mm}$. Assuming an average varus joint line inclination of $3^{\circ}$, when the mLDFA approaches $90^{\circ}$, the EG increases by $1.5 \mathrm{~mm}$, and when the mLDFA is $85^{\circ}$, the EG decreases by $1.5 \mathrm{~mm}$. Considering MPTA, although it is correlated with bone resection amount to a certain degree, its impact is so weak that it can be ignored.

Patients in subgroup 3 had valgus knees with varus tibias and accounted for the majority of the valgus knees. Only MPTA has been shown to be related to the extent of bone resection. In contrast with mLDFA in subgroup 1, MPTA had a weaker influence on the bone resection amount, and both the regression coefficient and $\mathrm{R}^{2}$ were smaller than those of mLDFA in subgroup 1 . The small number of patients in subgroup 3 might contribute to this observation.

The accuracy of the simulated bone cut was verified by intraoperative caliper bone resection in this study. Although only 10 patients were enrolled for intraoperative verification in this study, each case had 4 numerical variables: medial femoral condyle, lateral femoral condyle, medial tibial plateau and lateral tibial plateau. Therefore, a total of 40 paired measurements were tested for intraoperative verification, meaning that the power for distinguishing a difference of 0.5 in ICC exceeds $90 \%$ [25]. The retrospective 
medical record review did show that the actual bone resection adjustment during surgery was correlated with the corresponding joint line parameters. Although data visualization can provide useful information, we found that not all bone resection adjustments occurred in subgroups 1 and 3 . This result revealed that the degree of operative error has a significant impact on the accuracy of bone resection.

Our study has several limitations. First, a small number of patients were included in our study. There may be some selection bias, as we excluded some patients due to rotation of the lower extremities in fulllength weight-bearing radiographs, and abnormal rotation of the lower extremities results in inaccurate measurement of the MPTA [26]. In addition, because of the limited sample size, it may be that not all patients diagnosed with knee OA can be classified into 4 subgroups. One study reported a valgus knee with an mLDFA as large as $92^{\circ}$ [27]. Second, some special types of extra-articular deformities, such as tibial or femoral shaft bowing with progression of knee OA, were not used as alignment parameters. Although tibial or femoral bowing could dramatically change the joint line orientation, such deformities rarely need correction during primary TKA, so our study did not focus on these special extra-articular deformities. Finally, full-length weight-bearing radiographs were used for radiographic measurement rather than 3D CT, and some studies have proven that the latter has higher accuracy [28]. However, the cost effectiveness of radiographs is much greater. As a routine before TKA, some meaningful findings in preoperative radiographs would be more helpful for guiding clinical practice.

\section{Conclusion}

Knee joint deformities in patients diagnosed with OA can be artificially divided into 4 subgroups based on extremity alignment and joint line orientation as follows: varus knees with $m L D F A<90^{\circ}$, varus knees with mLDFA $>90^{\circ}$, valgus knees with MPTA $<90^{\circ}$ and valgus knees with MPTA $>90^{\circ}$. Patients in subgroup 1 accounted for the majority of all patients $(61.3 \%)$ in the current case series. When a TKA is performed on a varus knee with $\mathrm{mLDFA}<90^{\circ}$ using conventional instruments and the MA technique, the amount of bone resection in the EG is linearly related to the patient's mLDFA; with every $2^{\circ}$ of increase in mLDFA, the amount of bone resected will increase by approximately $1 \mathrm{~mm}$.

\section{Abbreviations}

TKA: Total knee arthroplasty; MA: Mechanical alignment; OA: Osteoarthritis; EG: Extension gap; PACS: Picture archiving and communication systems; HKA: Hip-knee-ankle; mLDFA: Mechanical lateral distal femoral angle; JLCA: Joint line congruency angle; MPTA: Medial proximal tibial angle

\section{Declarations}

\section{Ethics approval and consent to participate}

This study was approved by the Ethic board of Beijing Chaoyang Hospital (2015-S-4). Written informed consents for receiving TKA were obtained from all patients. This written informed consent includes 
surgical benefits, possible complications, and medical images and data related to surgical procedure may be used for further clinical studies.

\section{Consent for publication}

Not applicable.

\section{Availability of data and materials}

All data generated or analyzed during this study are included in this published article and its supplementary information files.

\section{Competing interests}

The authors declare that they have no competing interests.

\section{Funding}

National Natural Science Foundation of China (81572180).

\section{Authors' contributions}

ZWW contributed to the study design, performed the surgical procedure, statistical interpretation and drafted the manuscript. YY and DSM performed radiographic measurement, collected raw data and retrospective review patients' medical records. LW performed the surgical procedures, intraoperative bone resection measurement and data analysis. TBQ contributed to the study design, gained ethics approval and clinical coordination. All authors have read and approved the final manuscript.

\section{Acknowledgements}

Not applicable

\section{References}

1. Fang DM, Ritter MA, Davis KE. Coronal alignment in total knee arthroplasty: just how important is it? The Journal of arthroplasty. 2009;24(6 Suppl):39-43. doi:10.1016/j.arth.2009.04.034.

2. Lombardi AV, Jr., Berend KR, Ng VY. Neutral mechanical alignment: a requirement for successful TKA: affirms. Orthopedics. 2011;34(9):e504-6. doi:10.3928/01477447-20110714-40.

3. Ritter MA, Davis KE, Meding JB, Pierson JL, Berend ME, Malinzak RA. The effect of alignment and $\mathrm{BMI}$ on failure of total knee replacement. The Journal of bone and joint surgery American volume. 2011;93(17):1588-96. doi:10.2106/JBJS.J.00772.

4. Berend ME, Ritter MA, Meding JB, Faris PM, Keating EM, Redelman R et al. The Chetranjan Ranawat Award: Tibial Component Failure Mechanisms in Total Knee Arthroplasty. Clinical orthopaedics and related research. 2004;428:26-34. doi:10.1097/01.blo.0000148578.22729.0e. 
5. Green GV, Berend KR, Berend ME, Glisson RR, Vail TP. The effects of varus tibial alignment on proximal tibial surface strain in total knee arthroplasty: The posteromedial hot spot. The Journal of arthroplasty. 2002;17(8):1033-9. doi:10.1054/arth.2002.35796.

6. Victor JM, Bassens D, Bellemans J, Gursu S, Dhollander AA, Verdonk PC. Constitutional varus does not affect joint line orientation in the coronal plane. Clinical orthopaedics and related research. 2014;472(1):98-104. doi:10.1007/s11999-013-2898-6.

7. Bellemans J, Colyn W, Vandenneucker H, Victor J. The Chitranjan Ranawat award: is neutral mechanical alignment normal for all patients? The concept of constitutional varus. Clinical orthopaedics and related research. 2012;470(1):45-53. doi:10.1007/s11999-011-1936-5.

8. Schnurr C, Csécsei G, Nessler J, Eysel P, König DP. How much tibial resection is required in total knee arthroplasty? International orthopaedics. 2010;35(7):989-94. doi:10.1007/s00264-010-1025-5.

9. Paley D, Herzenberg JE, Tetsworth K, McKie J, Bhave A. Deformity planning for frontal and sagittal plane corrective osteotomies. The Orthopedic clinics of North America. 1994;25(3):425-65.

10. Tan H, Wang Y, Long T, Nie B, Mao Z, Yue B. How to accurately determine the distal femoral valgus cut angle in the valgus knee arthroplasty. International orthopaedics. 2018;42(3):537-42. doi:10.1007/s00264-018-3778-1.

11. Lombardi AV, Berend KR, Ng VY. Neutral mechanical alignment: a requirement for successful TKA: affirms. Orthopedics. 2011;32(9):691-.

12. Schiraldi M, Bonzanini G, Chirillo D, de Tullio V. Mechanical and kinematic alignment in total knee arthroplasty. Annals of translational medicine. 2016;4(7):130. doi:10.21037/atm.2016.03.31.

13. Abdel MP, Oussedik S, Parratte S, Lustig S, Haddad FS. Coronal alignment in total knee replacement: historical review, contemporary analysis, and future direction. The bone \& joint journal. 2014;96B(7):857-62. doi:10.1302/0301-620X.96B7.33946.

14. Rivière C, Iranpour F, Auvinet E, Aframian A, Asare K, Harris S et al. Mechanical alignment technique for TKA: Are there intrinsic technical limitations? Orthopaedics \& Traumatology: Surgery \& Research. 2017;103(7):1057-67. doi:10.1016/j.otsr.2017.06.017.

15. Hess S, Moser LB, Amsler F, Behrend H, Hirschmann MT. Highly variable coronal tibial and femoral alignment in osteoarthritic knees: a systematic review. Knee surgery, sports traumatology, arthroscopy : official journal of the ESSKA. 2019;27(5):1368-77. doi:10.1007/s00167-019-05506-2.

16. Rand J. Preoperative planning for total knee arthroplasty. Orthopedic knowledge update Hip and knee reconstruction American Academy of Orthopedic Surgeons, Rosemont, IL. 1995.

17. Yoshii I, Whiteside LA, White SE, Milliano MT. Influence of prosthetic joint line position on knee kinematics and patellar position. The Journal of arthroplasty. 1991;6(2):169-77.

18. Zeng YM, Wang Y, Zhu ZA, Dai KR. Effects of sex and lower extremity alignment on orientation of the knee joint line in knee surgery. Chinese medical journal. 2012;125(12):2126-31.

19. Hirschmann MT, Moser LB, Amsler F, Behrend H, Leclerq V, Hess S. Functional knee phenotypes: a novel classification for phenotyping the coronal lower limb alignment based on the native alignment 
in young non-osteoarthritic patients. Knee surgery, sports traumatology, arthroscopy : official journal of the ESSKA. 2019;27(5):1394-402. doi:10.1007/s00167-019-05509-z.

20. Lin YH, Chang FS, Chen KH, Huang KC, Su KC. Mismatch between femur and tibia coronal alignment in the knee joint: classification of five lower limb types according to femoral and tibial mechanical alignment. BMC musculoskeletal disorders. 2018;19(1):411. doi:10.1186/s12891-018-2335-9.

21. Rossi R, Rosso F, Cottino U, Dettoni F, Bonasia DE, Bruzzone M. Total knee arthroplasty in the valgus knee. International orthopaedics. 2014;38(2):273-83. doi:10.1007/s00264-013-2227-4.

22. Matsumoto T, Hashimura M, Takayama K, Ishida K, Kawakami Y, Matsuzaki T et al. A radiographic analysis of alignment of the lower extremities-initiation and progression of varus-type knee osteoarthritis. Osteoarthritis and cartilage. 2015;23(2):217-23. doi:10.1016/j.joca.2014.11.015.

23. Kim JM, Hong SH, Kim JM, Lee BS, Kim DE, Kim KA et al. Femoral shaft bowing in the coronal plane has more significant effect on the coronal alignment of TKA than proximal or distal variations of femoral shape. Knee surgery, sports traumatology, arthroscopy : official journal of the ESSKA. 2015;23(7):1936-42. doi:10.1007/s00167-014-3006-5.

24. Bellemans J, Vandenneucker $\mathrm{H}$, Vanlauwe J, Victor J. The influence of coronal plane deformity on mediolateral ligament status: an observational study in varus knees. Knee surgery, sports traumatology, arthroscopy : official journal of the ESSKA. 2010;18(2):152-6. doi:10.1007/s00167009-0903-0.

25. Bujang MA, Baharum N. A simplified guide to determination of sample size requirements for estimating the value of intraclass correlation coefficient: a review. Archives of Orofacial Science. 2017;12(1).

26. Jamali AA, Meehan JP, Moroski NM, Anderson MJ, Lamba R, Parise C. Do small changes in rotation affect measurements of lower extremity limb alignment? Journal of orthopaedic surgery and research. 2017;12(1):77. doi:10.1186/s13018-017-0571-6.

27. Eberbach H, Mehl J, Feucht MJ, Bode G, Sudkamp NP, Niemeyer P. Geometry of the Valgus Knee: Contradicting the Dogma of a Femoral-Based Deformity. The American journal of sports medicine. 2017;45(4):909-14. doi:10.1177/0363546516676266.

28. Eckhoff DG, Bach JM, Spitzer VM, Reinig KD, Bagur MM, Baldini TH et al. Three-dimensional mechanics, kinematics, and morphology of the knee viewed in virtual reality. The Journal of bone and joint surgery American volume. 2005;87 Suppl 2:71-80. doi:10.2106/JBJS.E.00440.

\section{Tables}

Table 1 Patients' demographics and radiographic joint line parameters 


\begin{tabular}{|c|c|}
\hline Number of patients & 112 \\
\hline Gender(male/female) & $25 / 87$ \\
\hline Age $\llbracket$ years old, mean $\pm \mathrm{SD} \square$ & $68.7 \pm 8.5$ \\
\hline Number of knees & 137 \\
\hline Side(left/right) & $67 / 70$ \\
\hline Deformity(varus/valgus) & $116 / 21$ \\
\hline $\mathrm{HKA}($ degree, mean $\pm \mathrm{SD})$ & $-5.6 \pm 6.2$ \\
\hline mLDFA(degree, mean \pm SD) & $88.1 \pm 2.9$ \\
\hline $\mathrm{JLCA}($ degree, mean $\pm \mathrm{SD})$ & $-3.6 \pm 3.1$ \\
\hline MPTA(degree, mean \pm SD) & $86.1 \pm 2.4$ \\
\hline Subgroup 1 (varus knee, $\operatorname{mLDFA}<90^{\circ}$ ) & $84(61.3 \%)$ \\
\hline Subgroup 2 (varus knee, $\mathrm{mLDFA}>90^{\circ}$ ) & $32(23.4 \%)$ \\
\hline Subgroup 3 (valgus knee, MPTA $<90^{\circ}$ ) & $14(10.2 \%)$ \\
\hline Subgroup 4 (valgus knee, MPTA $>90^{\circ}$ ) & $7(5.1 \%)$ \\
\hline
\end{tabular}

Table 2. Intraoperative adjustment of primary bone cut in different subgroups of patients

\begin{tabular}{lllllll}
\hline & $-2 \mathrm{f}$ & $-2 \mathrm{t}$ & 0 & $+2 \mathrm{f}$ & $+2 \mathrm{t}$ & +4 \\
\hline Subgroup1 & 0 & 1 & 59 & 6 & 14 & 4 \\
Subgroup2 & 0 & 4 & 24 & 1 & 1 & 2 \\
Subgroup3 & 0 & 1 & 10 & 2 & 1 & 0 \\
Subgroup4 & 2 & 0 & 4 & 0 & 1 & 0 \\
Total(n, \%) & $2(1.5 \%)$ & $6(4.4 \%)$ & $97(70.8 \%)$ & $9(6.6 \%)$ & $17(12.4 \%)$ & $6(4.4 \%)$ \\
\hline
\end{tabular}

\section{Figures}




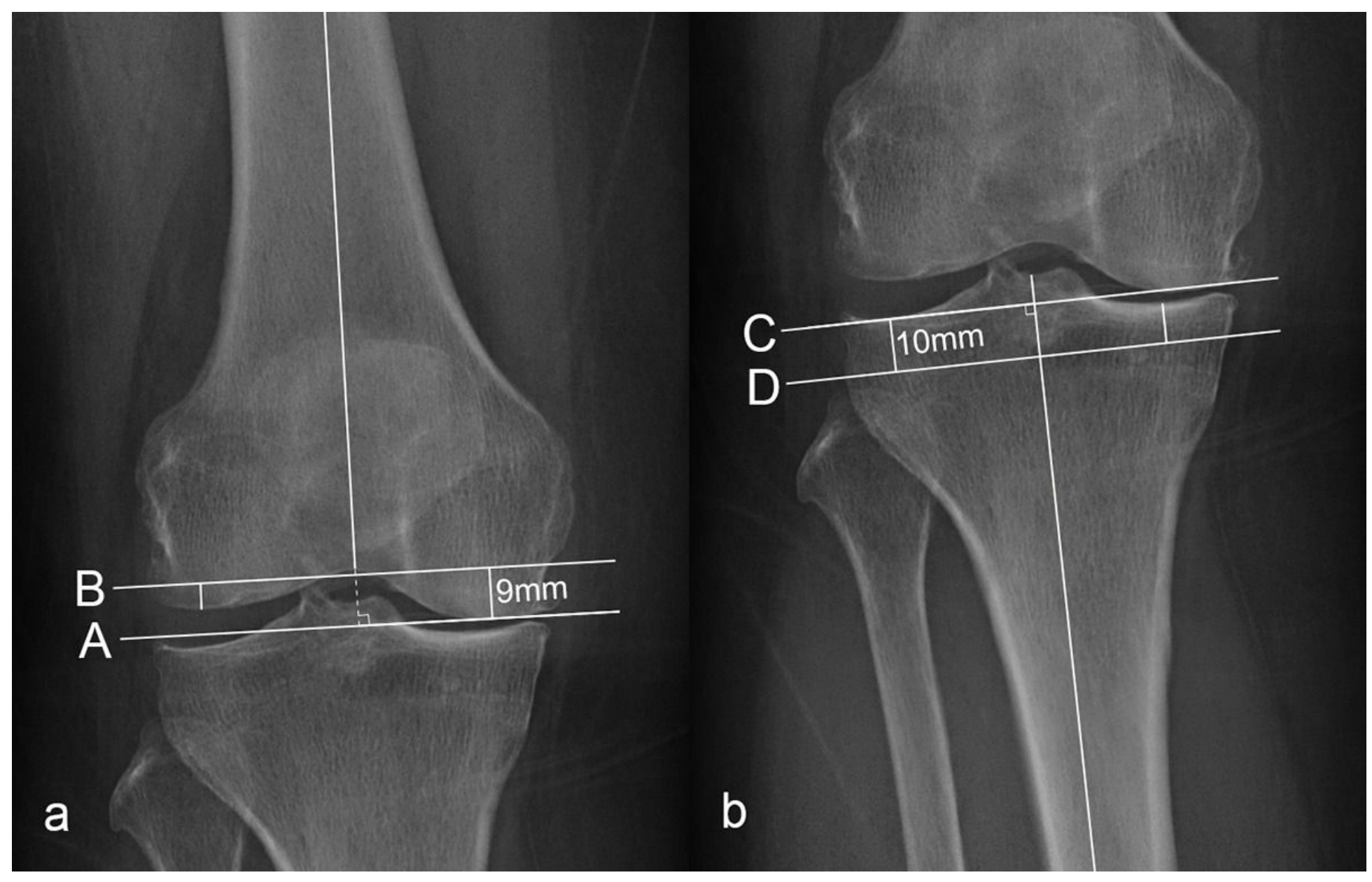

\section{Figure 1}

Partial schematics of simulated bone resection in full-length weight bearing radiograph. The bone resection thicknesses the femoral condyles (a) and tibial plateau (b) are shown. Line $A$ is tangential to the most distal femur and perpendicular to femoral mechanical axis, Line $B$ is parallel to Line $A$ and is $9 \mathrm{~mm}$ away from Line $A$. Line $C$ is tangential to the most proximal articular surface and perpendicular to tibial mechanical axis, Line $\mathrm{D}$ is parallel to Line $\mathrm{C}$ and $10 \mathrm{~mm}$ below Line $\mathrm{C}$. 




\section{Figure 2}

Demonstration of the resected bone pieces in TKA (a) and the measurement of the thickness of the resections with a caliper (b). In case of intact cartilage, $-2 \mathrm{~mm}$ of measured value; oscillating saw kerf $(1.5 \mathrm{~mm})$ should be compensated in all resections. 


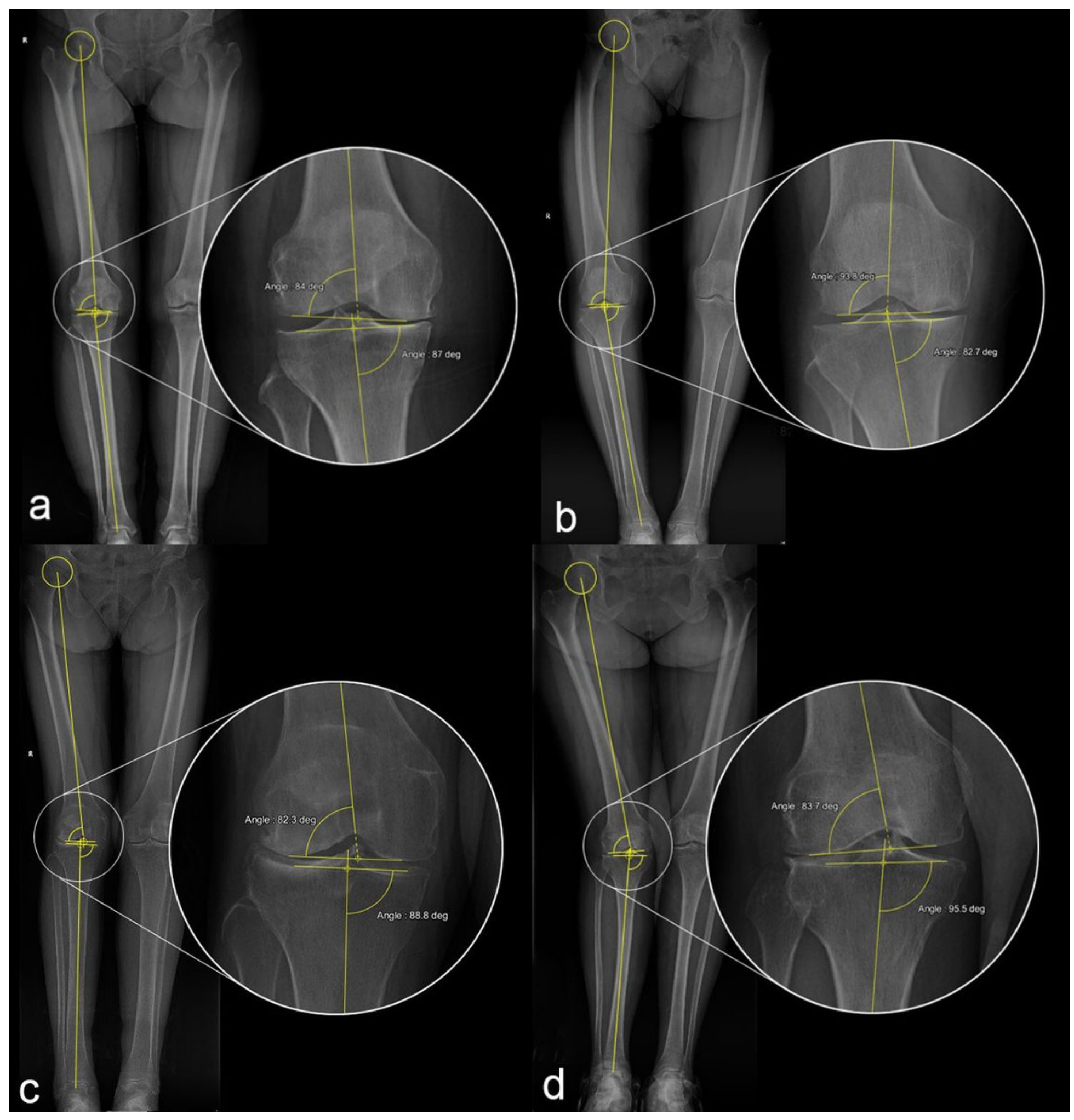

\section{Figure 3}

Patients can be divided into 4 subgroups based on limb alignment and joint orientation: (a) varus knee with $\mathrm{mLDFA}<90^{\circ}$, (b) varus knee with mLDFA $>90^{\circ}$, (c) valgus knee with MPTA $<90^{\circ}$ and (d) valgus knee with MPTA $>90^{\circ}$. 




Figure 4

The relationship between Max. resection and joint orientation parameters in varus knee with mLDFA $<90$ degrees. Scatter dots weighted by Max. resection are shown. Compared with less bone cuts (green dots) in the bottom left quadrant, more bone cuts (red dots) appear in the upper quadrant rather than the most right quadrant. This shows that mLDFA has more impact on the amount of bone cut.

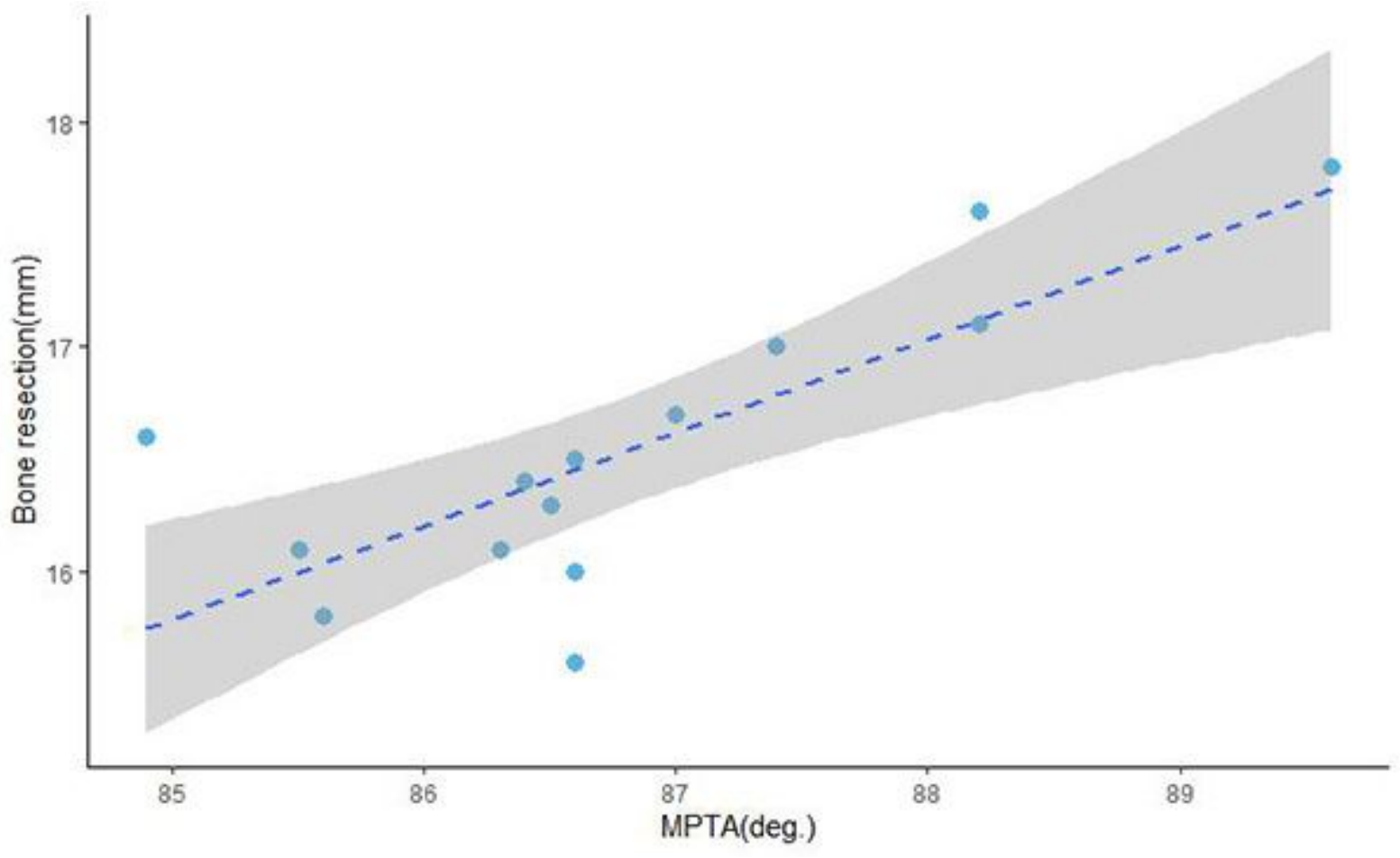

Figure 5 
Linear regression plot of Max. resection and MPTA in valgus knee with mLDFA $<90$ degrees. The shaded part in the plot is the $95 \%$ confidence interval.


Figure 6

The relationship between intraoperative adjustment of bone cut and mLDFA in subgroup 1 (a), and MPTA in subgroup 3 (b). Note: 0 no bone cut adjustment, $+2 \mathrm{t} 2 \mathrm{~mm}$ of tibial recut, $+2 \mathrm{f} 2 \mathrm{~mm}$ of distal femoral recut, $+42 \mathrm{~mm}$ of tibial and $2 \mathrm{~mm}$ of femoral recut, $-2 \mathrm{t}$ applied $2 \mathrm{~mm}$ thicker liner. 